\title{
SÍNDROME DE AICARDI: VARIABILIDAD FENOTÍPICA Y FACTORES PRONÓSTICOS
}

\section{CLINICAL OUTCOME OF DISTINCT AICARDI SYNDROME PHENOTYPES}

\author{
GALDÓS M ${ }^{1}$, MARTÍNEZ R ${ }^{1}$, PRATS JM²
}

\section{RESUMEN}

Objetivo: Analizar y comparar la casuística de nuestro hospital con los casos descritos en la literatura de Síndrome de Aicardi. El Síndrome de Aicardi es una rara enfermedad genética que afecta a las mujeres, y se caracteriza por agenesia del cuerpo calloso asociada a otras malformaciones del sistema nervioso central y lagunas coriorretinianas.

Métodos: Se presentan tres casos clínicos de Síndrome de Aicardi, con importantes diferencias fenotípicas y de evolución de la enfermedad.

Resultados: El primer caso es el caso más benigno de Síndrome de Aicardi descrito en la literatura, y se caracteriza por la baja morbilidad ocular, ausencia de epilepsia y de anomalías de la migración neuronal y desarrollo psicomotor normal. El segundo caso presenta larga supervivencia y pocas alteraciones oculares, pero un retardo severo en el desarrollo psicomotor. El tercer caso presenta alteraciones oculares más severas y mala evolución clínica con mortalidad a una edad temprana.

Conclusiones: El Síndrome de Aicardi puede ser fenotípicamente heterogéneo presentando gran variabilidad en la severidad de características clíni-

\begin{abstract}
Objective: Three cases of Aicardi Syndrome were diagnosed in our hospital. This syndrome is a rare, female-restricted genetic disease, characterized by agenesis of the corpus callosum, other central nervous system malformations, and chorioretinal lacunae. We have compared these cases with other cases of Aicardi Syndrome described in the world literature.

Methods: We have reported the three cases of Aicardi Syndrome and detailed the important heterogeneity of phenotypic features and clinical severity.

Results: The most benign case (case number 1) was characterized by mild ocular morbidity, absence of both migration abnormalities and epilepsy, and normal psychomotor development. Case number 2 achieved long-term survival with mild ocular alterations, but had severe retardation in psychomotor development. Case number 3 had the most severe ocular abnormalities which evolved rapidly and resulted in early death.

Conclusions: Aicardi Syndrome can be phenotypically heterogeneous, presenting with substantial variability in the severity of clinical features such as psychomotor development and survival. Our study
\end{abstract}

\footnotetext{
Recibido: 11/5/06. Aceptado: 15/11/07.

Departamento de Oftalmología Pediátrica y Departamento de Neurología Pediátrica. Hospital de Cruces. Vizcaya. España.

1 Licenciado en Medicina.

2 Doctor en Medicina.

Comunicación presentada inicialmente en el LXXX Congreso de la S.E.O. (Córdoba 2004).

Correspondencia:

Marta Galdós

Dpto. de Oftalmología. Hospital de Cruces

Plaza de Cruces, s/n

48903 Baracaldo (Vizcaya)

España

E-mail: martagaldos@monteurquiola.com
} 
cas como el desarrollo psicomotor y el pronóstico de supervivencia. Nuestro estudio apoyaría que las anomalías de la migración neuronal y las lesiones coriorretinianas pueden ser factores pronósticos de esta enfermedad.

Palabras clave: Síndrome de Aicardi, lagunas corioretinianas, anomalías de la migración neuronal, epilepsia, coloboma, desarrollo psicomotor. indicates that cortical migration abnormalities and retinal lesions may be useful prognostic factors (Arch Soc Esp Oftalmol 2008; 83: 29-36).

Key words: Aicardi Syndrome, chorioretinal lacunae, cortical migration abnormalities, epilepsy, coloboma, psychomotor development.

\section{INTRODUCCIÓN}

El síndrome de Aicardi es una afectación congénita poco frecuente y en general muy severa, que se caracteriza típicamente por la agenesia del cuerpo calloso, espasmos infantiles y lagunas coriorretinianas en el fondo de ojo. Las complejas malformaciones del sistema nervioso central pueden incluir defectos de la migración neuronal, así como malformaciones quísticas, que pueden causar convulsiones y retardo mental severo.

El síndrome de Aicardi engloba manifestaciones oculares, que incluyen colobomas a nivel papilar y lagunas coriorretinianas. Otras manifestaciones sistémicas pueden presentarse en el síndrome de Aicardi, como las anomalías costovertebrales y la displasia renal.

El pronóstico infausto asociado clásicamente al síndrome de Aicardi parece tener cada vez más matices y excepciones, con una pequeña proporción de niñas afectadas que presentan un retardo mental sólo leve o moderado (1). Sin embargo, todavía hoy, no está claro qué características del síndrome se correlacionan con un buen pronóstico en términos de desarrollo psicomotor y de supervivencia. En el presente artículo, se describen tres casos de síndrome de Aicardi, cada uno con distinta expresión clínica y fenotípica. Nuestras observaciones apuntan algunas características fenotípicas que podrían sentar la base para dar un pronóstico más preciso a los pacientes con síndrome de Aicardi.

\section{SUJETOS, MATERIAL Y MÉTODOS}

\section{Caso 1}

Niña de 3 años y 8 meses de edad. Diabetes gestacional y madre $\mathrm{Rh}$ - grado A, gestación de 38 semanas y parto normal. Cariotipo femenino normal 46 XX.
El diagnóstico por ecografía prenatal de esta niña muestra agenesia del cuerpo calloso y riñón derecho displásico multiquístico. En el sistema nervioso central presenta un quiste de plexos coroideos y un quiste en la región interhemisférica profunda. En el examen de resonancia magnética nuclear, no se detectan anomalías de la migración neuronal (fig. 1). Los registros electroencefalográficos (EEG) fueron normales. Los estudios cognitivos muestran un cociente de desarrollo psicomotor normal.

La exploración del fondo de ojo muestra una apariencia prácticamente normal del fondo de ojo derecho, con una papila óptica normal, dos pequeñas lagunas coriorretinianas hipopigmentadas y ausencia de afectación macular. Sin embargo, observamos en el fondo de ojo izquierdo una malformación colobomatosa del nervio óptico y lagunas coriorretinianas en mayor número sin afectación macular (fig. 2).

\section{Caso 2}

Niña de 11 años y 10 meses de edad. Cariotipo femenino normal, gestación y parto normal. La resonancia magnética nuclear cerebral (RMN) muestra agenesia de cuerpo calloso junto con extensas anomalías de la migración neuronal (fig. 3). Esta paciente presenta epilepsia en la infancia siendo los primeros episodios de convulsiones a los 2 años de edad. Los registros electroencefalográficos (EEG), demuestran una hipsarritmia asimétrica. En el examen funduscópico aparece una papila completa en el ojo derecho, pigmento peripapilar y áreas de lagunas coriorretinianas hipopigmentadas peripapilares que se extienden hacia las arcadas vasculares retinianas. Sin embargo, no hay afectación macular. El fondo de ojo izquierdo, presenta un disco óptico colobomatoso y pequeñas áreas hipopigmentadas coriorretinales. No presenta otras 

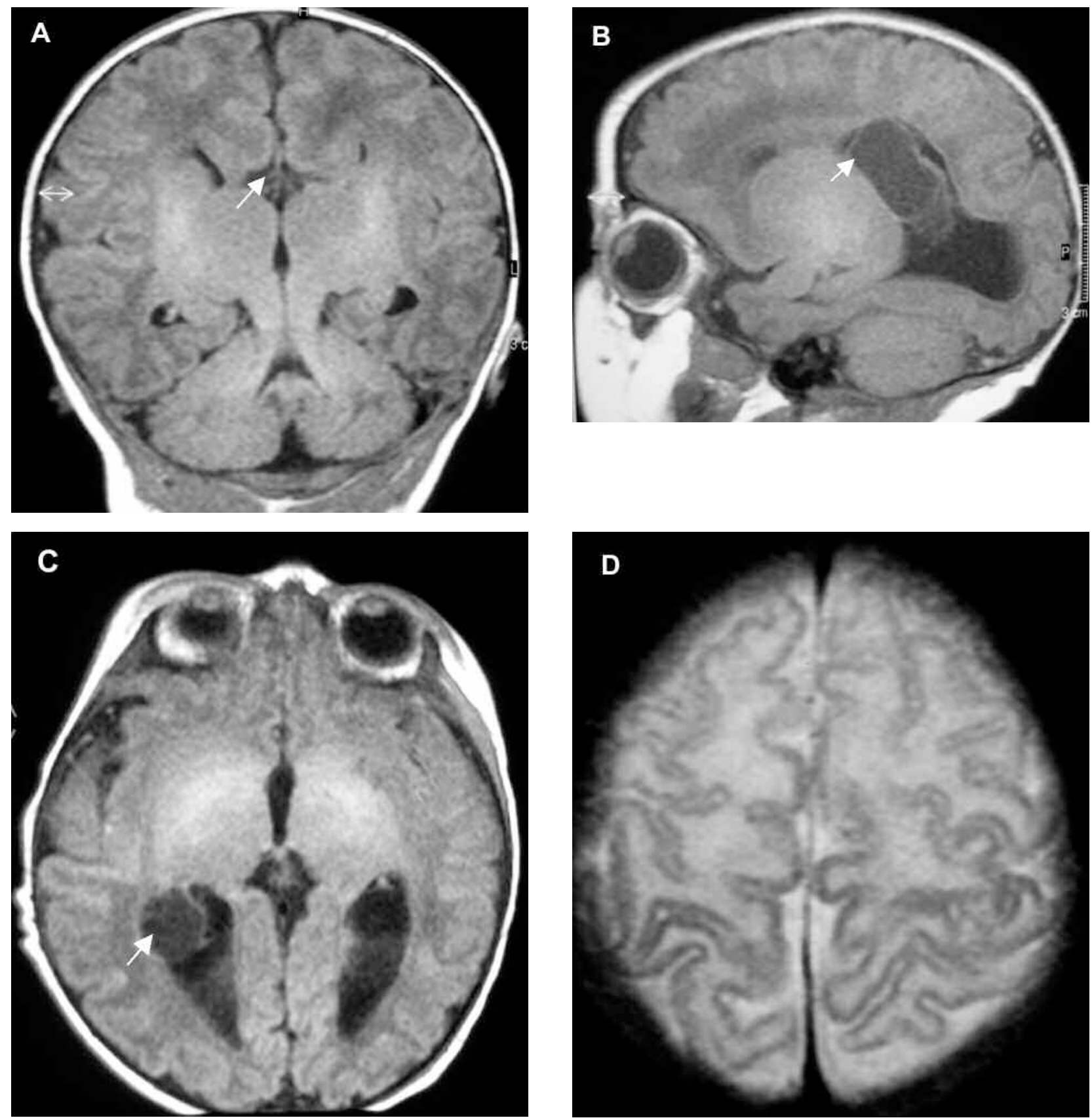

Fig. 1: RMN cerebral del paciente 1 con Síndrome de Aicardi. (A) Corte coronal en T1 muestra agenesia de cuerpo calloso (flecha). (B) Corte sagital en $T 1$ muestra un quiste subaracnoideo (flecha) en región interhemisférica profunda. (C) Corte axial en T1 ilustrando un quiste de plexos coroideos (flecha). (D) Corte axial en T2 muestra la ausencia macroscópica de malformaciones cerebrales compatibles con anomalías de la migración neuronal.

malformaciones a nivel renal o vertebral. La paciente se caracteriza por su larga supervivencia, con una edad actual de 11 años y 10 meses, pero presentando un retardo psicomotor muy severo.

\section{Caso 3}

La tercera paciente presenta el fenotipo más severo de nuestra casuística de Síndrome de Aicar- 


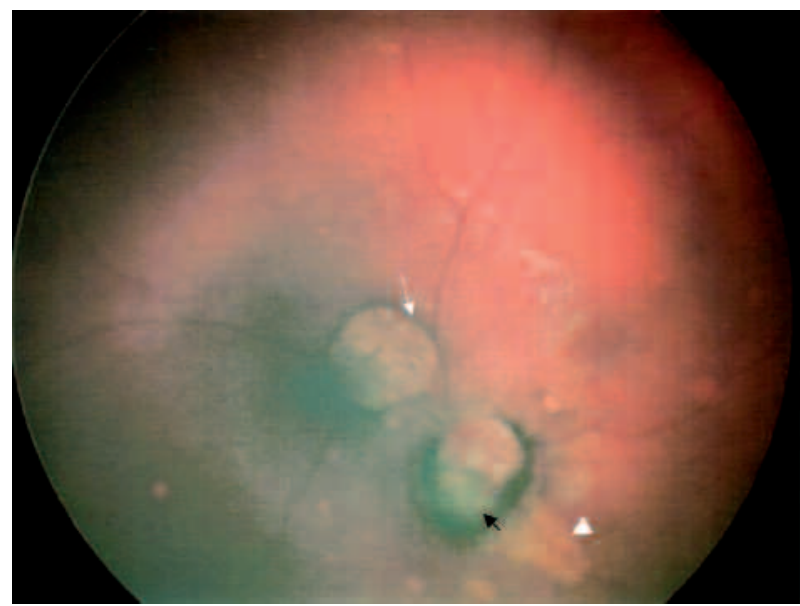

Fig. 2: Fondo de ojo izquierdo de la Paciente 1. Coloboma peripapilar nasal (flecha negra), pigmentación peripapilar y laguna coriorretiniana hipopigmentada superior a papila (flecha blanca) con pigmento perilacunar. Observamos otra laguna hipopigmentada de menor tamaño inferior a papila (punta de flecha) así como otras pequeñas lagunas coriorretinianas agrupadas alrededor de la papila y cerca de las arcadas vasculares. No hay afectación de la retina central ni del área macular. Nota: No es posible documentar más imágenes funduscópicas de los otros casos, por no disponer de retinógrafo portátil y fallecimiento precoz.

di. Gestación normal a término, parto normal, cariotipo femenino normal.

La resonancia magnética nuclear cerebral (RMN) se realiza a los 4 días de edad mostrando una malformación compleja con agenesia de cuerpo calloso, el lóbulo frontal izquierdo displástico con heterotopias nodulares subcorticales, quiste aracnoideo en fosa posterior, y coloboma en globo ocular izquierdo (fig. 4). La paciente muestra importante afectación en el desarrollo psicomotor. En el EEG se observa una hipsarritmia asimétrica muy desorganizada, presentando clínicamente crisis convulsivas refractarias al tratamiento.

El ojo izquierdo presenta una microftalmía importante. En la exploración del fondo de ojo derecho se observa un disco óptico derecho colobomatoso tipo Morning-Glory, con lagunas coriorretinianas en retina central, afectando el área macular. El fondo de ojo izquierdo incluye una papila colobomatosa tipo Morning-Glory con lagunas coriorretinianas circundantes. A los 9 meses de edad, la paciente presenta un desprendimiento de retina bulloso inferior en el ojo izquierdo. Los potenciales
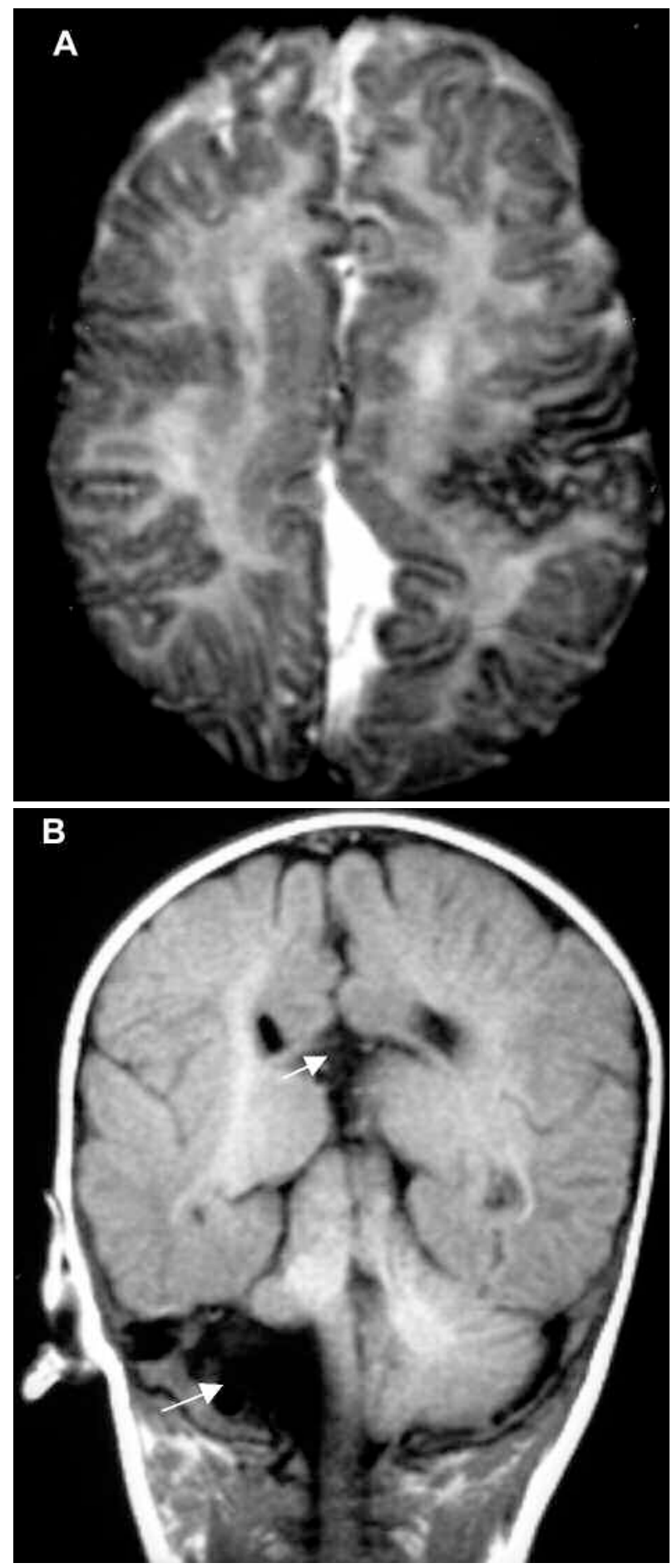

Fig. 3: RMN cerebral de la Paciente 2 con Síndrome de Aicardi. (A) Corte axial en T2 mostrando importantes anomalías de la migración cortical, con zonas de polimicrogiria principalmente en el hemisferio cerebral izquierdo. (B) Corte coronal en T1 muestra agenesia de cuerpo calloso (flecha superior) y agenesia del lóbulo derecho del cerebelo (flecha inferior). 

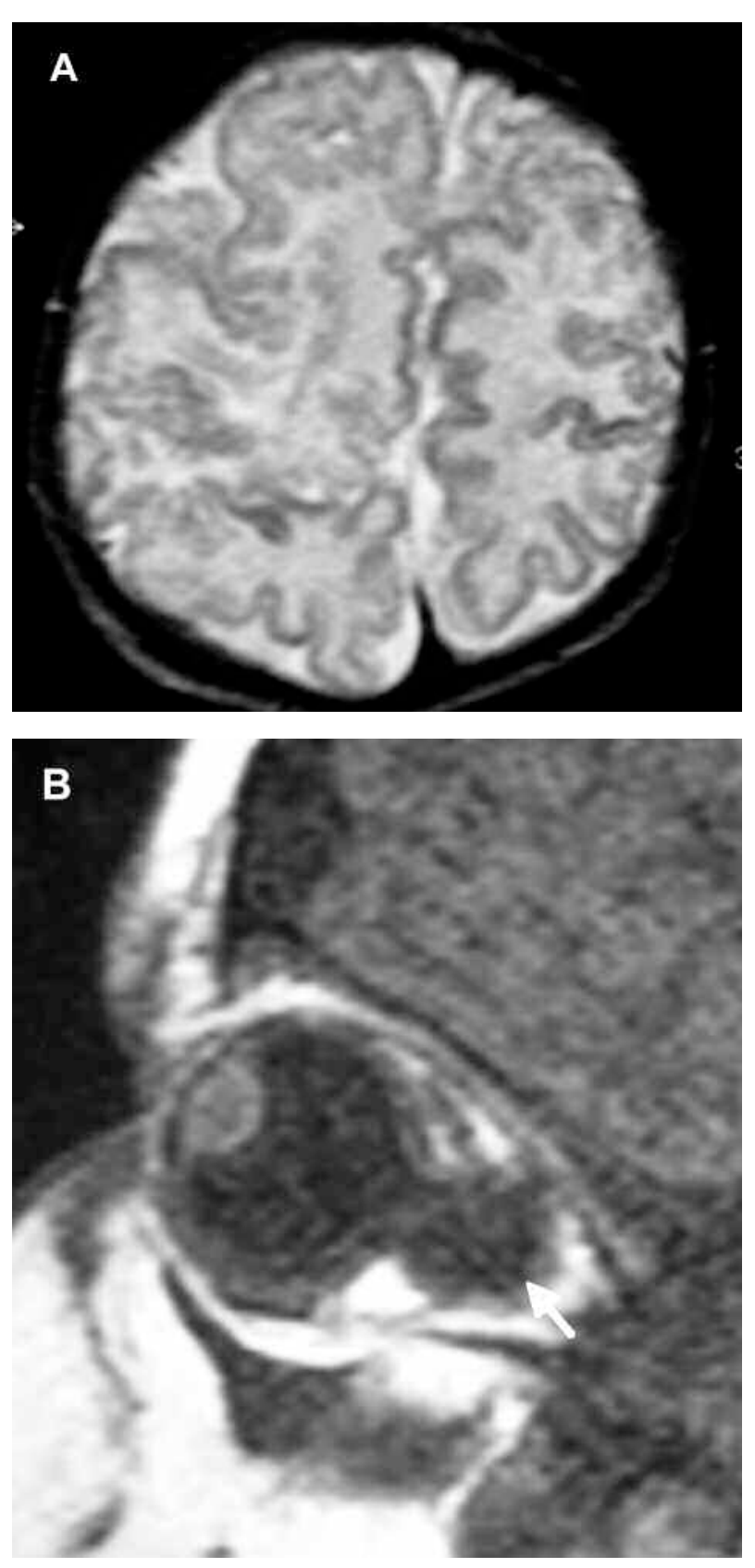

Fig. 4: RMN cerebral de la Paciente 3 con Síndrome de Aicardi. (A) Corte axial en T2 muestra importantes anomalías de la migración a nivel cortical. (B) Gran coloboma en el globo ocular izquierdo (flecha).

evocados visuales (PEV) están retrasados en el ojo derecho y ausentes en el ojo izquierdo. La paciente presenta también agenesia renal izquierda, hemivértebras y escoliosis severa. La paciente fallece a los 18 meses de edad.

\section{RESULTADOS (tabla I)}

El primer caso es el más benigno, presentando a los 3 años y 8 meses de edad ausencia de crisis, buen desarrollo visual, y desarrollo psicomotor normal. En el sistema nervioso central, se caracteriza por no presentar anomalías corticales ni de la de la migración neuronal. En el ojo presenta islotes lacunares de pequeño tamaño en fondo de ojo, con respeto macular y pequeño coloboma de nervio óptico unilateral. No presenta complicaciones tipo desprendimiento de retina, y está en ortoforia con buen desarrollo visual.

La segunda paciente presenta retardo psicomotor severo, y epilepsia severa con mal control clínico. Sin embargo se caracteriza por la buena supervivencia, con una edad actual de 11 años y 10 meses. En el sistema nervioso central, observan alteraciones corticales de la migración neuronal. Presenta benignidad en la clínica ocular, con lesiones coriorretinianas pequeñas, con respeto macular y pequeño coloboma de nervio óptico unilateral. Tampoco presenta complicaciones oculares asociadas, y está en ortoforia con buen desarrollo visual. No asocia otras malformaciones renales o vertebrales.

La tercera paciente es el caso más severo, con epilepsia refractaria y profundo retardo psicomotor, falleciendo a los 18 meses de edad. En la corteza presenta importantes anomalías de la migración neuronal. Las alteraciones oculares son severas con PEV patológicos, observando en el fondo de ojo grandes lagunas coriorretinianas con afectación del área macular, coloboma bilateral de nervio óptico de gran tamaño, ojo izquierdo microftálmico y evolucion a desprendimiento de retina. Asimismo presenta malformaciones sistémicas más severas con agenesia renal y múltiples alteraciones vertebrales.

\section{DISCUSIÓN}

El Síndrome de Aicardi puede ser fenotípicamente heterogéneo, presentando una variabilidad sustancial en la severidad de sus manifestaciones clínicas, en el desarrollo psicomotor y en la supervivencia (1-4). La serie más larga publicada en los últimos veinte años es de 77 casos miembros de la Fundación Aicardi y procedentes de todo el mundo. Se recogió información de los cuestionarios realizados a los familiares, refiriendo la presencia de retraso mental significativo en todos los casos, y con- 
Tabla I. Resultados: características clínicas con potencial valor pronóstico y su relación con la evolución clínica de tres pacientes con síndrome de Aicardi

Paciente 1

CORTEZA CEREBRAL

- Anomalías corticales de la migración neuronal

- Epilepsia

FONDO DE OJO

- Tamaño de lagunas cr

- Afectación macular de lagunas cr

- Coloboma de nervio óptico/tamaño

- Desprendimiento de retina

EVOLUCIÓN

- Desarrollo psicomotor

- Supervivencia

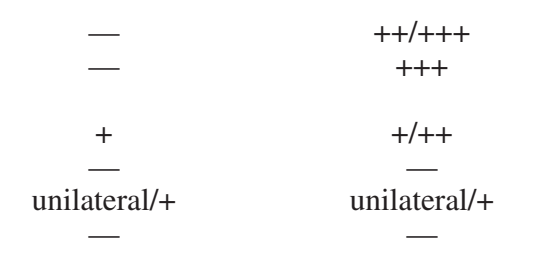

Normal 3 años 8 meses

Paciente 2

Paciente 3

Lagunas cr, lagunas coriorretinianas; (-), ausencia de esta característica clínica; (+), (++), (+++), lesion leve/moderada/ severa.

vulsiones en un $92 \%$ de los casos (5). Una reciente publicación analiza las respuestas de los cuidadores de 69 pacientes con Síndrome de Aicardi, aportando nuevos datos sobre la edad media de supervivencia estimada en 18,5 años, más favorable que lo publicado previamente (6). Pocos estudios han examinado las características clínicas que pueden predecir la evolución clínica $(7,8)$.

En este estudio, se presentan tres casos de Síndrome de Aicardi que representan una gradación clínica fenotípica (de leve a severa) y su pronóstico evolutivo. Hemos encontrado una asociación de algunas características específicas encefálicas y oculares con un mejor pronóstico clínico, apoyando artículos previos sobre el potencial valor pronóstico de características clínicas específicas $(7,8)$ cuya detección pueda contribuir a dar un pronóstico más preciso a los pacientes afectos de este síndrome.

En el cerebro, la ausencia de anomalías corticales de la migración neuronal es un factor de buen pronóstico en cuanto al desarrollo psicomotor y la supervivencia (caso 1, paciente que no presenta epilepsia y cuyo desarrollo psicomotor es normal).

En el ojo, la afectación unilateral del coloboma de nervio óptico y la presencia de lagunas coriorretinanas predominantemente de pequeño tamaño y con respeto de la retina central y de la mácula, también se relacionan con un mejor pronóstico en términos de desarrollo psicomotor (caso 1) y de supervivencia (casos 1 y 2) así como de buen desarrollo visual (casos 1 y 2). Esto ya había sido considerado previamente como factor de buen pronóstico en términos de desarrollo psicomotor y del lenguaje, así como de buen desarrollo de la función visual (8).
La paciente 1 parece ser el caso más benigno de Síndrome de Aicardi descrito en la literatura hasta la fecha $(3,4)$. Curiosamente, la baja morbilidad ocular (coloboma unilateral, lagunas coriorretinianas predominantemente de pequeño tamaño con respeto macular) se relaciona con un buen desarrollo psicomotor y buen estado general (ausencia de epilepsia), a pesar de presentar importantes malformaciones en el sistema nervioso central.

Asimismo, observamos también que la presencia de un coloboma unilateral, junto con el respeto macular de las lagunas coriorretinianas, son características clínicas que aparecen también en el caso 2 , relacionándose con una buena supervivencia en presencia de malformaciones cerebrales complejas junto con epilepsia, y severo retardo psicomotor (edad actual 11 años y 10 meses).

Por el contrario, encontramos como factores de mal pronóstico de supervivencia y de mayor severidad clínica la magnitud y la bilateralidad de lesiones de disco óptico de tipo colobomatoso, así como fosetas de disco óptico que puedan originar desprendimientos de retina serosos y el mayor tamaño de las lagunas coriorretinianas con afectación de la retina central y macular (paciente 3 que presentó una gran morbilidad clínica, gran retardo psicomotor y epilepsia severa sobreviviendo 18 meses).

El estudio de fondo de ojo cuidadoso y detallado puede no sólo tener un valor diagnóstico del Síndrome de Aicardi, sino también una significación pronóstica. Basándonos en estas observaciones consideramos que un buen control oftalmológico es obligado en estos pacientes.

Por último, entendemos que se necesitan series más largas de casos para validar los resultados que 
hemos obtenido. Nosotros hemos analizado nuestra casuística que resulta altamente sugestiva.

\section{BIBLIOGRAFÍA}

1. Aicardi J. Aicardi syndrome. Brain Dev 2005; 27: 164171.

2. Matlary A, Prescott T, Tvedt B, Lindberg K, Server A, Aicardi J, et al. Aicardi syndrome with mild developmental delay, absence of epilepsy and normal EEG. Clin Dysmorphol 2004; 13: 257-260.

3. Prats Vinas JM, Martinez Gonzalez MJ, Garcia Ribes A, Martinez Gonzalez S, Martinez Fernandez R. Callosal agenesis, chorioretinal lacunae, absence of infantile spasms, and normal development: Aicardi syndrome without epilepsy? Dev Med Child Neurol 2005; 47: 419-420.
4. Aicardi J. Callosal agenesis, chorioretinal lacunae, absence of infantile spasms and normal development: Aicardi syndrome without epilepsy? Dev Med Child Neurol 2005; 47: 364.

5. Rosser TL, Acosta MT, Packer RJ. Aicardi syndrome: spectrum of disease and long-term prognosis in $77 \mathrm{fema}$ les. Pediatr Neurol 2002; 27: 343-346.

6. Glasmacher MA, Sutton VR, Hopkins B, Eble T, Lewis RA, Park Parsons D, et al. Phenotiype and management of Aicardi syndrome: new findings from a survey of 69 children. J Child Neurol 2007; 22: 176-184.

7. Yacoub M, Missaoui N, Tabarli B, Ghorbel M, Tlili K, Selmi $\mathrm{H}$, et al. Aicardi syndrome with favourable outcome. Arch Pediatr 2003; 10: 530-532.

8. Menezes AV, Lewis TL, Buncic JR. Role of ocular involvement in the prediction of visual development and clinical prognosis in Aicardi syndrome. Br J Ophtalmol 1996; 80: 805-811. 